TRANSACTIONS OF THE

AMERICAN MATHEMATICAL SOCIETY

Volume 364, Number 12, December 2012, Pages 6671-6687

S 0002-9947(2012)05677-7

Article electronically published on June 12, 2012

\title{
ASYMPTOTICAL STABILITY OF MUCKENHOUPT WEIGHTS THROUGH GUROV-RESHETNYAK CLASSES
}

\author{
DANIEL AALTO AND LAURI BERKOVITS
}

\begin{abstract}
We show that weights in the Gurov-Reshetnyak class $G R_{\varepsilon}(\mu)$ satisfy a weak reverse Hölder inequality with an explicit and asymptotically sharp bound for the exponent, thus extending classical results from the Euclidean setting to doubling metric measure spaces. As an application, we study asymptotical behaviour of embeddings between Muckenhoupt classes and reverse Hölder classes.
\end{abstract}

\section{INTRODUCTION}

Muckenhoupt's weights are used extensively in harmonic analysis and the theory of partial differential equations. They serve as a natural substitute for the Lebesgue measure in the Euclidean space, since these weights span a rich enough realm sufficient to be applied in physical problems. They are fundamental in understanding the interplay of maximal operators and $L^{p}$-functions as well as quasiconformal mappings and functions of bounded mean oscillation; see [10].

In 1975, L. G. Gurov and Yu. G. Reshetnyak [12, 13 introduced another class of weights, defined in terms of mean oscillation. This class was used in the study of deformations and has found several applications. Surprisingly, the union of all the Gurov-Reshetnyak weights coincides with the largest of the Muckenhoupt's classes, $A_{\infty}$ [20].

Weights are not only useful in Euclidean spaces but also in more general metric spaces. Here we will consider a metric space equipped with a doubling measure $\mu$, with the weights studied against this underlying measure. We ask how Muckenhoupt's weights are related to Gurov-Reshetnyak weights and what is its connection to reverse Hölder inequalities. Moreover, we study the behaviour of weights under tightening conditions. As the defining constants are set to the strictest possible, the only remaining weights are constants. Hence, approaching these limits should ameliorate the properties of the weights themselves, and this is indeed the case.

In a doubling metric measure space the Euclidean results on Muckenhoupt's weights and related classes mostly hold; see [22] and [33]. It follows from the examples in the literature that the Gurov-Reshetnyak class is larger than the Muckenhoupt class $A_{\infty}$ and that a Gurov-Reshetnyak weight need not even be doubling 22]. However, we show that for small defining constants also Gurov-Reshetnyak weights are doubling.

Our results rely on a weak reverse Hölder inequality for Gurov-Reshetnyak weights. To obtain it, we use standard tools of analysis on metric spaces including Whitney type coverings, smooth partitions of unity and a Calderón-Zygmund type

Received by the editors May 12, 2011.

2010 Mathematics Subject Classification. Primary 42B25, 30L99.

(C) 2012 American Mathematical Society Reverts to public domain 28 years from publication 
decomposition. We got inspired by the works of A. A. Korenovski, A Lerner and A. Stokolos 20] and B. Bojarski [3. As an application of the main theorem, we investigate stability of Muckenhoupt classes and reverse Hölder inequalities. In particular, we show how knowledge of Gurov-Reshetnyak classes may be used to provide information about their asymptotical behaviour. Stability questions have been studied in many research articles; see, for instance, [3, 16, 17, 18, 21, 29, 32, 34, 35. Our approach to the subject seems to give some new results even in $\mathbb{R}^{n}$; see the remarks at the end of the last section.

The paper is organized as follows. Sections 2 and 3 contain mainly background information. The statement of the main result (Theorem 3.1) can be found in subsection 3.1 and is proved in sections 4 and 5 . In the remaining sections 6-8 consequences of the main result are studied.

\section{Doubling Metric MeAsure Spaces}

Throughout the paper $X=(X, d, \mu)$ is a metric space endowed with a metric $d$ and a Borel regular doubling measure $\mu$. An open ball always comes with a center and a positive finite radius, that is,

$$
B=B(x, r)=\{y \in X: d(y, x)<r\} .
$$

We denote by $\lambda B$ the $\lambda$-dilate of $B$, that is, a ball with the same center as $B$ but $\lambda$ times its radius. We assume that open balls have positive and finite measure. The doubling condition means there exists a constant $c_{\mu} \geq 1$, called the doubling constant of $\mu$, such that for all balls $B$ in $X$ we have

$$
\mu(2 B) \leq c_{\mu} \mu(B) .
$$

The doubling condition implies the important geometric property that a ball $B$ can only contain a limited number of pairwise disjoint balls of size comparable to that of $B$. This implies a covering argument, often referred to as Vitali's covering theorem: given a collection of balls with uniformly bounded radii, there exists a pairwise disjoint, countable subcollection of balls whose 5-dilates cover the union of the original collection. We shall use Vitali's theorem several times. First, we shall prove auxiliary Lemma 4.1 below and, second, construct Whitney type coverings, where bounded open sets are covered by balls with bounded overlap. For these results and more background on doubling metric measure spaces, we refer to monographs [6] and 33 .

\section{VARious Weight Classes}

We are interested in various different classes of non-negative, locally integrable functions (weights). Here we list the relevant definitions and some basic properties of them which will be needed in the sequel. Given a weight $w$ and a measurable set $E \subseteq X$, we often denote the $w$-measure by $w(E)=\int_{E} w \mathrm{~d} \mu$. For averages we use the notation

$$
w_{E}=f_{E} w \mathrm{~d} \mu=\frac{1}{\mu(E)} \int_{E} w \mathrm{~d} \mu .
$$

A weight is said to be doubling if it defines a doubling measure, that is, $w(2 B) \leq$ $c w(B)$ for all balls $B$. 
3.1. Gurov-Reshetnyak classes $G R_{\varepsilon}(\mu)$. We say that a weight $w$ belongs to $\operatorname{GR}_{\varepsilon}(\mu)$, where $0<\varepsilon<2$, if

$$
\left(G R_{\varepsilon}(\mu)\right) \quad f_{B}\left|w-w_{B}\right| \mathrm{d} \mu \leq \varepsilon w_{B}
$$

for all balls $B \subseteq X$. It should be observed that the condition is trivial for $\varepsilon$ larger than 2. A basic result in $\mathbb{R}^{n}$ equipped with the Lebesgue measure is that if the above condition holds for all subcubes $Q$ of a fixed cube $Q_{0}$ instead of balls, it implies higher integrability for $w$. Moreover, the order of integrability increases as $\varepsilon$ decreases. This was already observed by Gurov and Reshetnyak [12, 13. Different proofs in one- and multidimensional situations for the Lebesgue measure and doubling measures have appeared in 3, 4, 8, 9, 16, 19, 34. Some arguments only work for $\varepsilon$ close to 0 . Finally, a very simple proof in $\mathbb{R}^{n}$, for any $\varepsilon \in(0,2)$ for arbitrary absolutely continuous measures, was obtained in 20. It shows that weights belonging to Gurov-Reshetnyak classes satisfy the reverse Hölder inequality with an explicit and asymptotically optimal bound $p(\varepsilon)$ for the exponent: $p(\varepsilon) \asymp$ $c(n) / \varepsilon$ as $\varepsilon \rightarrow 0$, meaning that the ratio of the two quantities tend to 1 as $\varepsilon \rightarrow 0$. Certain generalizations of Gurov-Reshetnyak classes have been studied in the metric setting by D. V. Isangulova [14, 15], but there are some geometric restrictions because of the argument used. Our main result is the following extension to the setting of doubling metric measure spaces without any additional structure.

Theorem 3.1. Assume $w \in G R_{\varepsilon}(\mu)$, where $0<\varepsilon<2$. Then, there exists $p(\varepsilon)>1$ such that whenever $1 \leq p<p(\varepsilon)$, we have

$$
\left(f_{B} w^{p} \mathrm{~d} \mu\right)^{1 / p} \leq C f_{2 B} w \mathrm{~d} \mu
$$

for all balls $B$ with a constant $C$ depending on $\varepsilon$ and $c_{\mu}$. In addition, if $\varepsilon<2 c_{\mu}^{-1}$, the weight $w$ is doubling and consequently satisfies (3.2) with equally sized balls on both sides of the inequality. Moreover, $p(\varepsilon) \asymp c / \varepsilon$, as $\varepsilon \rightarrow 0$, where the constant $c$ only depends on the doubling constant of the underlying measure $\mu$.

It should be observed that the order we obtain, $p(\varepsilon) \asymp c / \varepsilon$, as $\varepsilon \rightarrow 0$, is the same as in the Euclidean setting, and therefore sharp; see [3, 8. In particular, this implies that as $\varepsilon \rightarrow \infty$, the admissible values of $p \rightarrow \infty$.

Throughout the paper, estimates of the form (3.2), where the right-hand side average is over the larger ball $2 B$, will be referred to as weak reverse Hölder inequalities.

3.2. Muckenhoupt classes $A_{p}(\mu)$. We say that a weight $w$ belongs to $A_{p}(\mu)$, where $1 \leq p<\infty$, if

$$
\left(A_{p}(\mu)\right) \quad f_{B} w \mathrm{~d} \mu \cdot\left(f_{B} w^{-1 /(p-1)} \mathrm{d} \mu\right)^{p-1} \leq c_{w}
$$

for all balls $B$ with a constant $c_{w} \geq 1$ independent of $B$. In $\mathbb{R}^{n}$ there are several equivalent descriptions of the class $A_{\infty}$, the union of all $A_{p}$ classes, but they are not all equivalent in the metric setting; see R. Korte and O. E. Kansanen [22]. In particular, if for some $\alpha, \beta \in(0,1)$ it holds that

$$
\mu\left(\left\{x \in B: w(x)>\beta w_{B}\right\}\right)>\alpha \mu(B),
$$


it does not follow that $w$ belong to any $A_{p}(\mu)$ for a finite $p$. Therefore, we cannot conclude the equivalence $\left(w \in A_{p}(\mu)\right.$ for some finite $p$ if and only if $w \in G R_{\varepsilon}(\mu)$ for some $\varepsilon$ ) between the $A_{p}(\mu)$ and $G R_{\varepsilon}(\mu)$ conditions as can be done in $\mathbb{R}^{n}$ for any absolutely continuous measure; see 20. However, all $A_{p}(\mu)$ weights satisfy (3.3), which allows us to conclude the $A_{p}(\mu) \Rightarrow G R_{\varepsilon}(\mu)$ part. Assuming a priori that the weight $w$ is doubling, the converse implication is true as well. This follows from Theorem 3.1 and characterizations of $A_{\infty}(\mu)$ in [22].

Our first application concerns the asymptotical behaviour of the $A_{1}(\mu)$ condition as the constant $c_{w}$ in the definition tends to one. We shall see that as $c_{w} \rightarrow 1$, the values of $p$ for which we have $w \in R H_{p}(\mu)$ tend to infinity.

3.3. Gehring classes $R H_{p}(\mu)$. The Gehring class or reverse Hölder class $R H_{p}(\mu)$, where $1<p \leq \infty$, consists of weights satisfying the inequality

$$
\left(f_{B} w^{p} \mathrm{~d} \mu\right)^{1 / p} \leq c_{w} f_{B} w \mathrm{~d} \mu
$$

for all balls $B \subseteq X$ with a constant $c_{w} \geq 1$ independent of $B$. These classes are connected to the Muckenhoupt classes in a similar way as the Gurov-Reshetnyak classes. In particular, in the metric setting $w \in R H_{p}(\mu)$ does not imply that the weight belong to any $A_{p}(\mu)$ for finite $p$ unless we assume a priori that the weight $w$ be doubling, as is demonstrated in $[22$.

Observe that for $p=\infty$ we have $\operatorname{ess} \sup _{B} w$ on the left-hand side of the reverse Hölder condition. The smallest reverse Hölder class $R H_{\infty}\left(\mathbb{R}^{n}\right)$ has been studied systematically by D. Cruz-Uribe and C. J. Neugebauer [7. Our second application deals with the asymptotical behaviour of the $R H_{\infty}(\mu)$ condition as $c_{w} \rightarrow 1$. It is dual to the result we had for $A_{1}(\mu)$ weights and shows that as $c_{w} \rightarrow 1$, the values of $p$ for which we have $w \in A_{p}(\mu)$ tend to one as well.

Finally, we consider Gehring's lemma, to which Theorem 3.1 gives a new proof (for weights $w$ having reverse Hölder constant $c_{w}$ close to 1) with the expected asymptotical behaviour. Namely, $w \in R H_{p}(\mu)$ implies $w \in R H_{q}(\mu)$ for some $q>p$, where the larger exponent $q \rightarrow \infty$ as $c_{w} \rightarrow 1$.

\section{Auxiliary Results}

To prove our main result we wish to adapt the approach of Korenovskyy et al. [20]. To begin with, we need a suitable substitute for the covering lemma in 26]. The following lemma can also be found in a previous paper by the first author [2], but we have included it here for completeness.

Lemma 4.1. Let $B_{0}$ be a ball, $F \subseteq 3 B_{0}, E=F \cap B_{0}$ and $\mu(F) \leq \rho \mu\left(B_{0}\right)$ with $0<\rho<1$. Then there exist a family $\left\{B_{i}\right\}_{i}$ of pairwise disjoint balls with $5 B_{i} \subseteq 3 B_{0}$ and

i) $\mu\left(E \backslash \bigcup_{i=1}^{\infty} 5 B_{i}\right)=0$,

ii) $\mu\left(5 B_{i} \cap F\right) \leq \frac{\rho}{1-\rho} \mu\left(5 B_{i} \backslash F\right)$,

iii) $\sum_{i=1}^{\infty} \mu\left(5 B_{i}\right) \leq \frac{c_{\mu}^{3}}{\rho} \mu(F)$.

Proof. Let $x \in E$ be a density point of $E$. Since

$$
\mu\left(F \cap B\left(x, 2 r_{0}\right)\right) \leq \mu(F) \leq \rho \mu\left(B_{0}\right) \leq \rho \mu\left(B\left(x, 2 r_{0}\right)\right),
$$


we have

$$
\mu\left(B\left(x, 2 r_{0}\right) \cap F\right) \leq \frac{\rho}{1-\rho} \mu\left(B\left(x, 2 r_{0}\right) \backslash F\right),
$$

and since $x$ is a density point of $E$, there exists the greatest integer $k$ so that $5 B_{x}=B\left(x, 2^{1-k} r_{0}\right)$ satisfies

$$
\mu\left(5 B_{x} \cap F\right) \leq \frac{\rho}{1-\rho} \mu\left(5 B_{x} \backslash F\right) .
$$

We apply Vitali's covering theorem to the balls $B_{x}$, each of which is associated with a density point $x$ of $E$. We obtain a countable family of pairwise disjoint balls $\left\{B_{i}\right\}$ satisfying condition ii). Since almost every point is a density point, condition i) also follows.

To see that iii) holds, we observe that the maximality of $k$ implies

$$
\mu\left(B\left(x, 2^{1-j} r_{0}\right) \cap F\right)>\frac{\rho}{1-\rho} \mu\left(B\left(x, 2^{1-j} r_{0}\right) \backslash F\right)
$$

for all $j>k$. Using this, we get

$$
\begin{aligned}
\sum_{i=1}^{\infty} \mu\left(5 B_{i}\right) & \leq c_{\mu}^{3} \sum_{i=1}^{\infty} \mu\left(\frac{5}{8} B_{i}\right) \\
& \leq \frac{c_{\mu}^{3}}{\rho} \sum_{i=1}^{\infty} \mu\left(\frac{5}{8} B_{i} \cap F\right) \\
& \leq \frac{c_{\mu}^{3}}{\rho} \mu(F) .
\end{aligned}
$$

The following two lemmas are given without proofs. Lemma 4.2 is due to Korenovskyy et al. [20]. Their paper also contains a converse result to the statement and therefore shows the aforementioned connection between Muckenhoupt classes and Gurov-Reshetnyak classes in $\mathbb{R}^{n}$. Lemma 4.3 is a well-known argument due to Muckenhoupt [28, a proof of which can also be found in [17].

Lemma 4.2. Assume $w \in G R_{\varepsilon}(\mu)$, where $0<\varepsilon<2$. Then, for $\varepsilon<\lambda<2$, we have

$$
\mu\left(\left\{x \in B: w(x)>(1-\varepsilon / \lambda) w_{B}\right\}\right) \geq(1-\lambda / 2) \mu(B) .
$$

Lemma 4.3. Assume $w \geq 0$ is a measurable function and let $B$ be a ball. Suppose that for all $s \geq s_{0} \geq 0$ and for some $c>1$ we have the estimate

$$
\int_{B \cap\{w>s\}} w \mathrm{~d} \mu \leq \operatorname{cs} \mu(B \cap\{w>s\}) .
$$

Then, whenever $1<p<c /(c-1)$, we have

$$
\int_{B \cap\left\{w>s_{0}\right\}} w^{p} \mathrm{~d} \mu \leq \frac{c}{c-p(c-1)} s_{0}^{p} \mu\left(B \cap\left\{w>s_{0}\right\}\right) .
$$

We need one more lemma. The proof makes use of standard arguments of theory of $A_{p}$ weights. (See [10, p. 405.) The upper bound $\varepsilon<2 c_{\mu}^{-1}$ in the lemma cannot be improved. Indeed, Example 4.1 given in 22. provides an example of a non-doubling weight $w \in G R_{1}(\mu)$, where the doubling constant of the underlying measure is $c_{\mu}=2$. 
Lemma 4.4. Assume $w \in G R_{\varepsilon}(\mu)$ with $\varepsilon<2 c_{\mu}^{-1}$. Then $w$ is a doubling weight.

Proof. Lemma 4.2 implies the existence of some $\alpha, \beta \in(0,1)$ such that for $S \subseteq B$, we have

$$
\frac{\mu(S)}{\mu(B)} \leq \alpha \Rightarrow \frac{w(S)}{w(B)} \leq \beta
$$

To prove the lemma we want to choose certain $\alpha$. Let us assume $w(S)>\beta w(B)$, where $\beta$ is to be chosen. Then $w(B \backslash S) \leq(1-\beta) w(B)$. Now write

$$
\begin{aligned}
B \backslash S= & \left\{x \in B \backslash S: w(x)>(1-\varepsilon / \lambda) w_{B}\right\} \\
& \cup\left\{x \in B \backslash S: w(x) \leq(1-\varepsilon / \lambda) w_{B}\right\} .
\end{aligned}
$$

From Lemma 4.2 and Chebyshev's inequality, we get

$$
\mu(B \backslash S) \leq\left(\frac{1-\beta}{1-\varepsilon / \lambda}+\frac{\lambda}{2}\right) \mu(B),
$$

giving

$$
\mu(S) \geq\left(1-\frac{1-\beta}{1-\varepsilon / \lambda}-\frac{\lambda}{2}\right) \mu(B) .
$$

The expression in the parentheses above will be $\alpha$. We wish to choose $\alpha:=1-c_{\mu}^{-1}$. To this end, we set $\beta:=1-\left(\frac{1}{c_{\mu}}-\frac{\lambda}{2}\right)\left(1-\frac{\varepsilon}{\lambda}\right) \in(0,1)$, once we have fixed any $\lambda \in\left(\varepsilon, 2 c_{\mu}^{-1}\right)$.

We now apply (4.5) to show that $w$ is a doubling weight. Clearly, we have

$$
\mu(2 B \backslash B) \leq \alpha \mu(2 B) .
$$

This implies $w(2 B \backslash B) \leq \beta w(2 B)$, and, consequently,

$$
w(2 B) \leq \frac{w(B)}{1-\beta} .
$$

\section{Proof of Theorem 3.1}

In this section we will prove the main theorem. The proof is divided into several steps. Whereas constants with subscripts have fixed values, constants $c$ and $C$ without subscripts are generic and depend only on the doubling constant $c_{\mu}$ of the underlying measure. We also want to point out that the several parameters involved here $(\rho, \lambda, \ldots)$ are there just to guarantee that the argument works for any $\varepsilon \in(0,2)$.

For technical reasons we shall formulate a localized version of the definition of Gurov-Reshetnyak classes as follows. Given a ball $B$, we write $w \in G R_{\varepsilon}(\mu, B)$ if the above Gurov-Reshetnyak condition $G R_{\varepsilon}(\mu)$ holds for all balls (of the space $X$ ) contained in $B$.

Lemma 5.1. Assume $w \in G R_{\varepsilon}(\mu, 3 B)$, where $0<\varepsilon<2$. Moreover, let $\varepsilon<\lambda<2$ and $\rho /(1-\rho)<1-\lambda / 2$. Then, for $s \geq \rho^{-1} c_{\mu}^{2} w_{3 B}$, we have

$$
\int_{B \cap\{w>s\}}(w-s) \mathrm{d} \mu \leq \frac{C}{\rho} \frac{\varepsilon \lambda s}{\lambda-\varepsilon} \mu(3 B \cap\{w>s\}) .
$$


Proof. Fix a ball $B_{0}$ and set

$$
\begin{aligned}
& F=\left\{x \in 3 B_{0}: w(x)>s\right\}, \\
& E=F \cap B_{0} .
\end{aligned}
$$

An application of Chebychev's inequality shows that $\mu(F) \leq \rho \mu\left(B_{0}\right)$ for all $s \geq$ $\rho^{-1} c_{\mu}^{2} w_{3 B_{0}}$, and we may apply Lemma 4.1. Let $\left\{B_{i}\right\}_{i}$ be the family of balls thus obtained.

By i) of the covering lemma, we get

$$
\begin{aligned}
& \int_{E}(w-s) \mathrm{d} \mu \leq \sum_{i=1}^{\infty} \int_{E \cap 5 B_{i}}(w-s) \mathrm{d} \mu \\
& \leq \sum_{i=1}^{\infty}\left(\int_{E \cap 5 B_{i}}\left|w-w_{5 B_{i}}\right| \mathrm{d} \mu+\mu\left(E \cap 5 B_{i}\right)\left(w_{5 B_{i}}-s\right)\right) .
\end{aligned}
$$

Let us estimate the second term in the parentheses above, We have, by ii) of Lemma 4.1 and the definition of $F$,

$$
\begin{aligned}
\mu\left(E \cap 5 B_{i}\right)\left(w_{5 B_{i}}-s\right) & \leq \frac{\rho}{1-\rho} \mu\left(5 B_{i} \backslash F\right)\left(w_{5 B_{i}}-s\right) \\
& \leq \int_{5 B_{i} \backslash F}\left(w_{5 B_{i}}-s\right) \mathrm{d} \mu \\
& \leq \int_{5 B_{i} \backslash E}\left|w-w_{5 B_{i}}\right| \mathrm{d} \mu .
\end{aligned}
$$

Now combine the previous estimates and use $w \in G R_{\varepsilon}\left(\mu, 3 B_{0}\right)$ to get

$$
\begin{aligned}
\int_{E}(w-s) \mathrm{d} \mu & \leq \sum_{i=1}^{\infty} \int_{5 B_{i}}\left|w-w_{5 B_{i}}\right| \mathrm{d} \mu \\
& \leq \varepsilon \sum_{i=1}^{\infty} \mu\left(5 B_{i}\right) w_{5 B_{i}} .
\end{aligned}
$$

By Lemma 4.2 and ii) of Lemma 4.1, we get

$$
\begin{aligned}
& \mu\left(\left\{x \in 5 B_{i}: w(x)>(1-\varepsilon / \lambda) w_{5 B_{i}}\right\}\right) \\
& \geq(1-\lambda / 2) \mu\left(5 B_{i}\right)>\frac{\rho}{1-\rho} \mu\left(5 B_{i}\right) \geq \mu\left(5 B_{i} \cap F\right) .
\end{aligned}
$$

Therefore, we have $(1-\varepsilon / \lambda) w_{5 B_{i}} \leq s$. From this and iii), we get

$$
\begin{aligned}
\int_{E}(w-s) \mathrm{d} \mu & \leq \varepsilon \sum_{i=1}^{\infty} \mu\left(5 B_{i}\right) \frac{s}{1-\varepsilon / \lambda} \\
& \leq \frac{c_{\mu}^{3}}{\rho} \frac{\varepsilon \lambda s}{\lambda-\varepsilon} \mu(F) .
\end{aligned}
$$

The reader should observe that our Lemma 5.1 is a metric counterpart of Theorem 2 in [20, although our result is much weaker since the distribution set on the right-hand side of (5.2) is contained in the larger ball $3 B$. For this reason Lemma 4.3 is not yet applicable. Let us view the essence of the forthcoming argument we use to overcome this obstacle. We can form a Whitney type covering for a ball $B$ 
by balls $B_{j}$ in such a way that $3 B_{j} \subseteq B$ and the balls $3 B_{j}$ have bounded overlap. Then we would like to argue

$$
\begin{aligned}
\int_{B \cap\{w>s\}}(w-s) \mathrm{d} \mu & \leq \sum_{j} \int_{B_{j} \cap\{w>s\}}(w-s) \mathrm{d} \mu \\
& \leq \sum_{j} C s \mu\left(3 B_{j} \cap\{w>s\}\right) \\
& \leq C s \mu(B \cap\{w>s\}) .
\end{aligned}
$$

But this doesn't work since the estimate (5.2) only applies to large $s \geq \rho^{-1} c_{\mu}^{2} w_{3 B_{j}}$, and there is no obvious way to control the averages $w_{3 B_{j}}$. Therefore, we replace $w \in G R_{\varepsilon}(\mu)$ by another function $\widetilde{w}$ which is locally Gurov-Reshetnyak and has the property that its averages over small balls are uniformly bounded; see Lemma 5.4 below. To this end, we shall construct an auxiliary weight function $v$ and then define $\widetilde{w}:=v w$. This is carried out in the next two lemmas.

Lemma 5.3. Let $B$ be a ball. Then there exists a function $v$, supported on $2 B$, such that, given $\varepsilon>0$, there is a $\delta>0$ with

i) $0 \leq v(x) \leq 1$ for all $x$ and $v(x) \geq c>0$ for all $x \in B$,

ii) $\sup _{\widetilde{B}} v-\inf _{\widetilde{B}} v \leq \varepsilon \inf _{\widetilde{B}} v$ whenever $\widetilde{B}$ is a ball with center $\widetilde{x} \in 2 B$ and radius $\widetilde{r} \leq \delta \operatorname{dist}(\widetilde{x}, X \backslash 2 B)$,

iii) $\sup _{\widetilde{B}} v \leq c_{1} \frac{\mu(\widetilde{B})}{\mu(2 B)}$ whenever $\widetilde{B}$ is a ball with center $\widetilde{x} \in 2 B$ and radius $\widetilde{r}=\frac{\delta}{2+\delta} \operatorname{dist}(\widetilde{x}, X \backslash 2 B)$. Here the constant $c_{1}$ depends on $\varepsilon$ and $c_{\mu}$.

Proof. Fix a ball $B_{0}$ and let $\varepsilon>0$ be arbitrary.

We shall form a Whitney type decomposition for the ball $2 B_{0}$. For each $x \in 2 B_{0}$, we associate the radius

$$
r_{x}=\frac{\operatorname{dist}\left(x, X \backslash 2 B_{0}\right)}{100} .
$$

Then apply Vitali's covering theorem for the balls $\frac{1}{15} B\left(x, r_{x}\right)$ to get a countable collection of balls $B_{k}=B\left(x_{k}, r_{k}\right)$ such that $2 B_{0}=\bigcup_{k} \frac{1}{3} B_{k}$ and the balls $\frac{1}{15} B_{k}$ are pairwise disjoint. We have the following properties:

1) $\frac{1}{2} r_{k} \leq r_{x} \leq 2 r_{k}$ whenever $x \in B_{k}$,

2) $\frac{1}{2} r_{k} \leq r_{k^{\prime}} \leq 2 r_{k}$ whenever $B_{k} \cap B_{k^{\prime}} \neq \emptyset$,

3) $c_{\mu}^{-2} \mu\left(B_{k}\right) \leq \mu\left(B_{k^{\prime}}\right) \leq c_{\mu}^{2} \mu\left(B_{k}\right)$ whenever $B_{k} \cap B_{k^{\prime}} \neq \emptyset$,

4) $\sum_{k} \chi_{B_{k}} \leq N$, and each $B_{k}$ intersects at most $N$ other balls $B_{k^{\prime}}$. Here $N$ is a constant depending only on the doubling constant $c_{\mu}$.

Now let $\left\{\psi_{k}\right\}_{k}$ be a partition of unity subordinate to the covering $\left\{B_{k}\right\}_{k}$ with every $\psi_{k}$ being Lipschitz with constant $L / r_{k}$, where $L$ only depends on the doubling constant of the measure. We have $\operatorname{spt}\left(\psi_{k}\right) \subseteq B_{k}$ and $\sum_{k} \psi_{k}=1$. The construction can be found, for instance, from a previous paper by the first author [1].

Let us define the auxiliary weight function by

$$
v(x):=\sum_{k} \frac{\mu\left(B_{k}\right)}{\mu\left(2 B_{0}\right)} \psi_{k}(x) .
$$

Observe that 4$)$ and $\operatorname{spt}\left(\psi_{k}\right) \subseteq B_{k}$ together imply that the sum if actually finite. 
We shall verify properties i)-iii). To start with, we notice that clearly $0 \leq v(x) \leq$ 1 for all $x$. To see that $v$ is bounded away from zero on $B_{0}$, take $x \in B_{0} \cap B_{k}$. Then

$$
r_{0} \leq \operatorname{dist}\left(x, X \backslash 2 B_{0}\right) \leq r_{k}+\operatorname{dist}\left(x_{k}, X \backslash 2 B_{0}\right)=101 r_{k},
$$

giving $\mu\left(2 B_{0}\right) \leq c_{\mu}^{9} \mu\left(B_{k}\right)$. Therefore, we have $v(x) \geq c_{\mu}^{-9}$ for all $x \in B_{0}$.

To verify condition ii), consider a ball $\widetilde{B}$ with center $\widetilde{x} \in 2 B_{0}$ and radius $\widetilde{r} \leq$ $\delta \operatorname{dist}\left(\widetilde{x}, X \backslash 2 B_{0}\right)$, where, say, $\delta \leq 10^{-4}$. Vitali's theorem then implies that $\widetilde{B} \subseteq B_{k_{0}}$ for some $k_{0}$. We consider the oscillation of $v$ on $\widetilde{B}$. For $x, y \in \widetilde{B}$, we have

$$
\begin{aligned}
v(x)-v(y) & \leq \sum_{k \in K} \frac{\mu\left(B_{k}\right)}{\mu\left(2 B_{0}\right)}\left|\psi_{k}(x)-\psi_{k}(y)\right| \\
& \leq \sum_{k \in K} \frac{\mu\left(B_{k}\right)}{\mu\left(2 B_{0}\right)} \frac{2 L \widetilde{r}}{r_{k}},
\end{aligned}
$$

where $k \in K$ if $B_{k}$ intersects $\widetilde{B}$. By 2) and 3), we have $r_{k_{0}} \leq 2 r_{k}$ and $\mu\left(B_{k}\right) \leq$ $c_{\mu}^{2} \mu\left(B_{k_{0}}\right)$ for every $k \in K$. Moreover, $\widetilde{r}=100 \delta r_{\widetilde{x}}$ and $r_{\widetilde{x}} \leq 2 r_{k_{0}}$. Hence, we get

$$
\begin{aligned}
\frac{\mu\left(B_{k}\right)}{\mu\left(2 B_{0}\right)} \frac{2 L \tilde{r}}{r_{k}} & \leq 4 L c_{\mu}^{2} \frac{\mu\left(B_{k_{0}}\right)}{\mu\left(2 B_{0}\right)} \frac{\tilde{r}}{r_{k_{0}}} \\
& \leq 800 c_{\mu}^{2} L \delta \frac{\mu\left(B_{k_{0}}\right)}{\mu\left(2 B_{0}\right)} .
\end{aligned}
$$

Further, by 3$)$ we have $\mu\left(B_{k_{0}}\right) \leq c_{\mu}^{2} \mu\left(B_{k}\right)$. This gives, for all $z \in \widetilde{B}$,

$$
\frac{\mu\left(B_{k_{0}}\right)}{\mu\left(2 B_{0}\right)}=\sum_{k \in K} \frac{\mu\left(B_{k_{0}}\right)}{\mu\left(2 B_{0}\right)} \psi_{k}(z) \leq c_{\mu}^{2} v(z) .
$$

Finally, combining the previous estimates and remembering 4), we conclude

$$
\sup _{\widetilde{B}} v-\inf _{\widetilde{B}} v \leq 800 c_{\mu}^{4} N L \delta \inf _{\widetilde{B}} v
$$

Now take $\delta:=\varepsilon /\left(800 c_{\mu}^{4} N L\right)$ to get ii).

Finally, we turn to iii) and take a ball $\widetilde{B}$ with center $\widetilde{x} \in 2 B_{0}$ and radius $\widetilde{r}=$ $\frac{\delta}{2+\delta} \operatorname{dist}\left(\widetilde{x}, X \backslash 2 B_{0}\right)$. Then it is contained in some $B_{k_{0}}$ and

$$
100 r_{k_{0}}=\operatorname{dist}\left(x_{k_{0}}, X \backslash 2 B_{0}\right) \leq r_{k_{0}}+(1+2 / \delta) \widetilde{r} .
$$

This gives $r_{k_{0}} \leq \widetilde{r} / \delta$, implying that $\mu\left(B_{k_{0}}\right) \leq C \mu(\widetilde{B})$ for some $C>0$. Taking into account 3$)$, we get from the property ii) that

$$
\sup _{\widetilde{B}} v \leq(1+\varepsilon) \inf _{\widetilde{B}} v \leq C \frac{\mu(\widetilde{B})}{\mu\left(2 B_{0}\right)} .
$$

Lemma 5.4. Let $B$ be a ball and assume $w \in G R_{\varepsilon_{0}}(\mu, 2 B)$, where $0<\varepsilon_{0}<2$. Then, given $\varepsilon_{1} \in\left(\varepsilon_{0}, 2\right)$, there exists a function $\widetilde{w}$ and $\gamma>0$ such that whenever $\widetilde{B}$ is a ball with center $\widetilde{x} \in 2 B$ and radius $\widetilde{r}=\gamma \operatorname{dist}(\widetilde{x}, X \backslash 2 B)$, we have

i) $0 \leq \widetilde{w}(x) \leq w(x)$ for all $x$ and $w(x) \leq c \widetilde{w}(x)$ for all $x \in B$,

ii) $\widetilde{w} \in G R_{\varepsilon_{1}}(\mu, \widetilde{B})$,

iii) $\widetilde{w}_{\widetilde{B}} \leq c_{1} w_{2 B}$. 
Proof. Fix a ball $B_{0}$. Let $v$ be the function given by the previous lemma. Then choose $\varepsilon$ such that $\varepsilon_{1}=(1+\varepsilon) \varepsilon_{0}+\varepsilon$ and apply the lemma to get the corresponding $\delta$. Now set $\widetilde{w}(x):=w(x) v(x)$ and $\gamma:=\delta /(2+\delta)$.

The properties in i) follow directly from those of $v$ in the corresponding part of the previous lemma. To prove ii), let $\widetilde{B}$ be a ball with center $\widetilde{x} \in 2 B_{0}$ and radius $\widetilde{r}=\gamma \operatorname{dist}\left(\widetilde{x}, X \backslash 2 B_{0}\right)$ and let $B=B(y, r)$ be any ball (of the space $X$ ) contained in $\widetilde{B}$. Then we may assume $r \leq 2 \widetilde{r}$ (for otherwise $B=\widetilde{B}$ ) and

$$
\widetilde{r} \leq \gamma \widetilde{r}+\gamma \operatorname{dist}\left(y, X \backslash 2 B_{0}\right),
$$

giving

$$
r \leq 2 \widetilde{r} \leq \frac{2 \gamma}{1-\gamma} \operatorname{dist}\left(y, X \backslash 2 B_{0}\right)=\delta \operatorname{dist}\left(y, X \backslash 2 B_{0}\right) .
$$

Thus, the requirements in part ii) of Lemma 5.3 are fulfilled. Denote

$$
B^{+}=\left\{x \in B: \widetilde{w}(x)-\widetilde{w}_{B}>0\right\}
$$

and $B^{-}=B \backslash B^{+}$. Moreover, set $S:=\sup _{B} v$ and $s:=\inf _{B} v$. Then, we have $s w \leq \widetilde{w} \leq S w$. By the estimate for the oscillation of $v$ in part ii) of Lemma 5.3 and the assumption $w \in G R_{\varepsilon_{0}}\left(\mu, 2 B_{0}\right)$, we get

$$
\begin{aligned}
\int_{B}\left|\widetilde{w}-\widetilde{w}_{B}\right| \mathrm{d} \mu & =\int_{B^{+}}\left(\widetilde{w}-\widetilde{w}_{B}\right) \mathrm{d} \mu-\int_{B^{-}}\left(\widetilde{w}-\widetilde{w}_{B}\right) \mathrm{d} \mu \\
& \leq \int_{B^{+}}\left(S w-s w_{B}\right) \mathrm{d} \mu-\int_{B^{-}}\left(s w-S w_{B}\right) \mathrm{d} \mu \\
& =(S-s) \int_{B} w \mathrm{~d} \mu+S \int_{B^{+}}\left(w-w_{B}\right) \mathrm{d} \mu-s \int_{B^{-}}\left(w-w_{B}\right) \mathrm{d} \mu \\
& \leq(S-s) \int_{B} w \mathrm{~d} \mu+S \int_{B}\left|w-w_{B}\right| \mathrm{d} \mu \\
& \leq \varepsilon \inf _{B} v \int_{B} w \mathrm{~d} \mu+\left(\sup _{B} v\right) \varepsilon_{0} \int_{B} w \mathrm{~d} \mu .
\end{aligned}
$$

Observe that $\sup _{B} v \leq(1+\varepsilon) \inf _{B} v$. Therefore, we get

$$
f_{B}\left|\widetilde{w}-\widetilde{w}_{B}\right| \mathrm{d} \mu \leq\left((1+\varepsilon) \varepsilon_{0}+\varepsilon\right) f_{B} \widetilde{w} \mathrm{~d} \mu .
$$

Consequently, $\widetilde{w} \in G R_{\varepsilon_{1}}(\mu, \widetilde{B})$.

It remains to prove iii). But this follows from the corresponding part of the previous lemma:

$$
f_{\widetilde{B}} \widetilde{w} \mathrm{~d} \mu \leq \frac{c_{1}}{\mu\left(2 B_{0}\right)} \int_{\widetilde{B}} \frac{\widetilde{w}}{\sup _{\widetilde{B}} v} \mathrm{~d} \mu \leq c_{1} w_{2 B_{0}} .
$$

We are now in a position to complete

Proof of Theorem 3.1. Let $w \in G R_{\varepsilon_{0}}(\mu)$, where $0<\varepsilon_{0}<2$. Let $\varepsilon_{0}<\lambda<2$ and $\rho /(1-\rho)<1-\lambda / 2$. Then, given a ball $B_{0}$, take $\varepsilon_{1} \in\left(\varepsilon_{0}, \lambda\right)$ and let $\widetilde{w}$ and $\gamma>0$ be as in the previous lemma. 
We shall form a Whitney type decomposition of $2 B_{0}$ with balls of appropriate size. For each $x \in 2 B_{0}$ we associate the radius

$$
r_{x}=\frac{\gamma \operatorname{dist}\left(x, X \backslash 2 B_{0}\right)}{15} .
$$

Then apply Vitali's covering theorem for the balls $B\left(x, r_{x}\right)$ to get a collection of balls $\widetilde{B}_{j}=B\left(\widetilde{x}_{j}, \widetilde{r}_{j}\right)$, where $\widetilde{r}_{j}=\gamma \operatorname{dist}\left(\widetilde{x}_{j}, X \backslash 2 B_{0}\right)$, with bounded overlap and $2 B_{0}=\bigcup_{j} \frac{1}{3} \widetilde{B}_{j}$.

By Lemma $5.4 \mathrm{ii}$ ), we have $\widetilde{w} \in G R_{\varepsilon_{1}}(\mu, \widetilde{B})$. By Lemma 5.1 we then have for $s \geq \rho^{-1} c_{\mu}^{2} \widetilde{w}_{\widetilde{B}_{j}}$,

$$
\int_{\frac{1}{3} \widetilde{B}_{j} \cap\{\widetilde{w}>s\}}(\widetilde{w}-s) \mathrm{d} \mu \leq \frac{c_{\mu}^{3}}{\rho} \frac{\varepsilon_{1} \lambda s}{\lambda-\varepsilon_{1}} \mu\left(\widetilde{B}_{j} \cap\{\widetilde{w}>s\}\right) .
$$

Then, by iii) in Lemma 5.4, for $s \geq s_{0}:=\rho^{-1} c_{\mu}^{2} c_{1} w_{2 B_{0}}$ we have

$$
\begin{aligned}
& \int_{2 B_{0} \cap\{\widetilde{w}>s\}}(\widetilde{w}-s) \mathrm{d} \mu \leq \sum_{j} \int_{\frac{1}{3} \widetilde{B}_{j} \cap\{\widetilde{w}>s\}}(\widetilde{w}-s) \mathrm{d} \mu \\
& \leq \sum_{j} \frac{c_{\mu}^{3}}{\rho} \frac{\varepsilon_{1} \lambda s}{\lambda-\varepsilon_{1}} \mu\left(\widetilde{B}_{j} \cap\{\widetilde{w}>s\}\right) \\
& \leq \frac{C}{\rho} \frac{\varepsilon_{1} \lambda s}{\lambda-\varepsilon_{1}} \mu\left(2 B_{0} \cap\{\widetilde{w}>s\}\right) .
\end{aligned}
$$

This implies

$$
\int_{2 B_{0} \cap\{\widetilde{w}>s\}} \widetilde{w} \mathrm{~d} \mu \leq\left(\frac{C}{\rho} \frac{\varepsilon_{1} \lambda}{\lambda-\varepsilon_{1}}+1\right) s \mu\left(2 B_{0} \cap\{\widetilde{w}>s\}\right) .
$$

Denote by $c_{0}$ the constant in the parentheses above. Then, whenever $1<p<$ $c_{0} /\left(c_{0}-1\right)$, we have, by Lemma 4.3 .

$$
\begin{aligned}
\int_{2 B_{0}} \widetilde{w}^{p} \mathrm{~d} \mu & =\int_{2 B_{0} \cap\left\{\widetilde{w} \leq s_{0}\right\}} \widetilde{w}^{p} \mathrm{~d} \mu+\int_{2 B_{0} \cap\left\{\widetilde{w}>s_{0}\right\}} \widetilde{w}^{p} \mathrm{~d} \mu \\
& \leq s_{0}^{p} \mu\left(2 B_{0} \cap\left\{\widetilde{w} \leq s_{0}\right\}\right)+C s_{0}^{p} \mu\left(2 B_{0} \cap\left\{\widetilde{w}>s_{0}\right\}\right) \\
& \leq C s_{0}^{p} \mu\left(2 B_{0}\right) .
\end{aligned}
$$

Finally, we use the fact that $w(x) \leq c \widetilde{w}(x)$ for every $x \in B_{0}$ and insert $s_{0}=$ $\rho^{-1} c_{\mu}^{2} c_{1} w_{2 B_{0}}$ to conclude

$$
f_{B_{0}} w^{p} \mathrm{~d} \mu \leq C f_{B_{0}} \widetilde{w}^{p} \mathrm{~d} \mu \leq C\left(f_{2 B_{0}} w \mathrm{~d} \mu\right)^{p}
$$

Having now seen that $w \in G R_{\varepsilon_{0}}(\mu)$ satisfies the weak reverse Hölder inequality (3.2), we may apply Lemma 4.4 to conclude that if $\varepsilon_{0}<2 c_{\mu}^{-1}$, then $w$ actually satisfies the inequality with averages over the same ball on both sides. Consequently, $w \in R H_{p}(\mu)$ for $1<p<c_{0} /\left(c_{0}-1\right)$. To see that we have the desired asymptotical behaviour as $\varepsilon \rightarrow 0$, we observe that if $\varepsilon$ is close 0 , we may choose, say, $\varepsilon_{1}=2 \varepsilon, \lambda=1$ and $\rho=1 / 4$. Then it it easy to see that the bound $p(\varepsilon)=1+c / \varepsilon$. 


\section{Embedding $A_{1}(\mu)$ into Reverse Hölder Classes}

Let $w$ be a weight satisfying the $A_{1}(\mu)$ condition of Muckenhoupt,

$$
\left(A_{1}(\mu)\right) \quad f_{B} w \mathrm{~d} \mu \leq c_{w} \underset{x \in B}{\operatorname{essinf}} w(x) .
$$

It is not difficult to see that if $c_{w}=1$, then $w$ is a constant function (up to a set of $\mu$-measure zero). Therefore, one might expect that as $c_{w} \rightarrow 1$, the order of local integrability of $w$ increases, and this (and even more) is indeed true: as $c_{w} \rightarrow 1$, the values of $p$ for which $w \in R H_{p}(\mu)$ tend to infinity. In the Euclidean setting this has been proved by B. Bojarski [3], by a method similar to ours, and by J. Kinnunen [17], using a different method. In the metric setting this can be derived as a simple application of our main result.

Lemma 6.1. If $w \in A_{1}(\mu)$ with constant $c_{w}$, then $w \in G R_{\varepsilon}(\mu)$, where

$$
\varepsilon=2\left(1-c_{w}^{-1}\right) .
$$

Proof. The $A_{1}(\mu)$ condition gives

$$
w(x) \geq \frac{w_{B}}{c_{w}} \text { for } \mu \text {-a.e. } x \in B .
$$

Therefore, we have

$$
f_{B}\left|w-w_{B}\right| \mathrm{d} \mu \leq 2 \cdot f_{B}\left(w-\frac{w_{B}}{c_{w}}\right) \mathrm{d} \mu=2\left(1-\frac{1}{c_{w}}\right) w_{B}
$$

Having seen that $A_{1}$ weights belong to Gurov-Reshetnyak classes with an $\varepsilon$ explicitely depending on the $A_{1}$ constant, we may apply Theorem 3.1 to prove the following embedding theorem.

Theorem 6.2. If $w \in A_{1}(\mu)$ with constant $c_{w}$, then there exists a number $p\left(c_{w}\right)>1$ such that $w \in R H_{p}(\mu)$ whenever $1<p<p\left(c_{w}\right)$. Moreover, we have $p\left(c_{w}\right) \rightarrow \infty$, as $c_{w} \rightarrow 1$.

Proof. From the previous lemma we know that $w \in G R_{\varepsilon}(\mu)$, where $\varepsilon=2\left(1-c_{w}^{-1}\right)$. Theorem 3.1 together with the fact that the measure $w \mathrm{~d} \mu$ is doubling gives

$$
\left(f_{B} w^{p} \mathrm{~d} \mu\right)^{1 / p} \leq C f_{2 B} w \mathrm{~d} \mu \leq C f_{B} w \mathrm{~d} \mu
$$

for $1<p<p(\varepsilon)=p\left(c_{w}\right)$. The dependence between $c_{w}$ and $\varepsilon$ implies the desired asymptotical behaviour. Indeed, if $c_{w}$ is close to 1 , we have $p\left(c_{w}\right)=1+c c_{w} /\left(c_{w}-1\right)$.

\section{Embedding $R H_{\infty}(\mu)$ Into Muckenhoupt Classes}

We have seen how knowledge of the Gurov-Reshetnyak classes can be used to obtain asymptotically sharp embedding of the Muckenhoupt class $A_{1}$ into the reverse Hölder classes. Here we will see that it works the other way around as well. If the $R H_{\infty}(\mu)$ constant $c_{w}=1$, then the weight is a constant function. Therefore, as $c_{w} \rightarrow 1$, it seems reasonable to expect that the values of $p$, for which we have 
$w \in A_{p}(\mu)$, tend to 1 . In $\mathbb{R}^{n}$ this has been proved by A. Politis [31] and independently by T. Mitsis [27, who both used knowledge of BMO. We shall apply our main result to see that it holds in the metric setting as well.

We recall that the $R H_{\infty}(\mu)$ condition is

$\left(R H_{\infty}(\mu)\right)$

$$
\underset{x \in B}{\operatorname{esssup}} w(x) \leq c_{w} f_{B} w \mathrm{~d} \mu .
$$

In the spirit of the classical $A_{p}$ theory, we interchange the roles of measures and begin by showing that if $w$ belongs to $R H_{\infty}(\mu)$, then $\frac{1}{w}$ satisfies the Gurov-Reshetnyak condition with respect to the measure $\mathrm{d} w=w \mathrm{~d} \mu$.

Lemma 7.1. If $w \in R H_{\infty}(\mu)$ with constant $c_{w}$, then $\frac{1}{w} \in G R_{\varepsilon}(\mathrm{d} w)$, where $\varepsilon=$ $2\left(1-c_{w}^{-1}\right)$.

Proof. The $R H_{\infty}(\mu)$ condition is readily seen to be equivalent to the fact that $\frac{1}{w} \in A_{1}(\mathrm{~d} w)$ with the same defining constant, that is,

$$
f_{B} \frac{1}{w} \mathrm{~d} w \leq c_{w} \underset{x \in B}{\operatorname{w}-\operatorname{essinf}} \frac{1}{w(x)} .
$$

The claim now follows from Lemma 6.1

There is an important technical detail to take into consideration before we can apply Theorem 3.1. The theorem only applies to doubling measures, while in the metric setting $w \in R H_{p}(\mu)$ does not necessarily imply that the measure $\mathrm{d} w=w \mathrm{~d} \mu$ be doubling. However, we are interested in the asymptotical beheviour of the $R H_{\infty}(\mu)$ condition. The next lemma shows that if the $R H_{\infty}(\mu)$ constant is close to 1 , then the weight must be doubling. In fact, we prove a more general result for later purposes.

Lemma 7.2. Assume $w \in R H_{p}(\mu), 1<p \leq \infty$, with constant

$$
c_{w}<\left(\frac{c_{\mu}}{c_{\mu}-1}\right)^{1 / p^{\prime}} .
$$

We recall that here and elsewhere, $p^{\prime}$ denotes the conjugate exponent of $p$. Then the weight $w$ is doubling with constant

$$
c_{d}=\frac{1}{1-c_{w}\left(1-c_{\mu}^{-1}\right)^{1 / p^{\prime}}} .
$$

Proof. Standard arguments (see [10]) give, for any ball $B$ and a measurable $S \subseteq B$,

$$
\frac{w(S)}{w(B)} \leq c_{w}\left(\frac{\mu(S)}{\mu(B)}\right)^{1 / p^{\prime}} .
$$

Replacing $B$ by $2 B$ and setting $S=2 B \backslash B$ gives

$$
\begin{aligned}
w(2 B \backslash B) & \leq c_{w}\left(\frac{\mu(2 B \backslash B)}{\mu(2 B)}\right)^{1 / p^{\prime}} w(2 B) \\
& \leq c_{w}\left(1-c_{\mu}^{-1}\right)^{1 / p^{\prime}} w(2 B),
\end{aligned}
$$


further giving

$$
w(2 B) \leq w(B)+c_{w}\left(1-c_{\mu}^{-1}\right)^{1 / p^{\prime}} w(2 B) .
$$

This in turn implies what we wanted.

We are ready to state and prove the main result of this section, the embedding theorem dual to Theorem 6.2.

Theorem 7.3. If $w \in R H_{\infty}(\mu)$ with constant $c_{w}<c_{\mu} /\left(c_{\mu}-1\right)$, then there is $p\left(c_{w}\right)>1$ such that $w \in A_{p}(\mu)$ whenever $p>p\left(c_{w}\right)$. Here $p\left(c_{w}\right) \rightarrow 1$, as $c_{w} \rightarrow 1$.

Proof. By the two previous lemmas, we have $\frac{1}{w} \in G R_{\varepsilon}(\mathrm{d} w)$, where $\varepsilon=2\left(1-c_{w}^{-1}\right)$ and the measure $\mathrm{d} w=w \mathrm{~d} \mu$ has doubling constant $c_{d}=\left(1-c_{w}+c_{w} / c_{\mu}\right)^{-1}$. By Theorem 3.1 we have

$$
\left(f_{B}\left(\frac{1}{w}\right)^{p^{\prime}} \mathrm{d} w\right)^{1 / p^{\prime}} \leq C f_{B} \frac{1}{w} \mathrm{~d} w
$$

for all $1<p^{\prime}<p^{\prime}(\varepsilon)=p^{\prime}\left(c_{w}\right)$. (Observe that " $B$ " instead of " $2 B$ " on the righthand side is obtained by a trivial estimate.) A simple rearranging shows that this is the $A_{p}(\mu)$ condition for $w$. We conclude that $w \in A_{p}(\mu)$ whenever $p>p\left(c_{w}\right)$. The dependence between the parameters $c_{w}, \varepsilon, p^{\prime}$ and $p$ implies that as the $R H_{\infty}(\mu)$ constant $c_{w} \rightarrow 1$, we have $p\left(c_{w}\right) \rightarrow 1$ as we wished. Precisely, we may choose $p\left(c_{w}\right)=1+c\left(c_{w}-1\right) / c_{w}$, provided $c_{w}$ is close to 1 .

\section{Asymptotical behaviour of Gehring's lemma}

Our third application is concerned with the asymptotical behaviour of the famous lemma due to F. W. Gehring [11]. The classical result states that if $w \in R H_{p}\left(\mathbb{R}^{n}\right)$, $1<p<\infty$, then $w \in R H_{q}\left(\mathbb{R}^{n}\right)$ for some $q>p$. It was observed by B. Bojarski [3] that as the reverse Hölder constant $c_{w} \rightarrow 1$, the larger exponent $q \rightarrow \infty$.

In the context of doubling metric measure spaces a weaker version of Gehring's lemma holds. It states that $w \in R H_{p}(\mu), 1<p<\infty$, implies that a weak reverse Hölder inequality with doubled ball on the right-hand side for a larger exponent holds; see, for instance, 23. We use Theorem 3.1 to prove Gehring's lemma in metric spaces for weights with reverse Hölder constant $c_{w}$ close to 1. Our argument yields an explicit bound for the larger exponent $q$ and shows that $q \rightarrow \infty$, as $c_{w} \rightarrow 1$. We argue as in the previous sections, but we have two cases $(p \in[2, \infty)$ or $p \in(1,2))$ to treat separately.

Lemma 8.1. If $w \in R H_{p}(\mu)$ with constant $c_{w}<\sqrt{5}$ and $p \geq 2$, then $w \in G R_{\varepsilon}(\mu)$, where $\varepsilon=\sqrt{c_{w}^{2}-1}$.

Proof. By Hölder's inequality and the assumptions, we have

$$
\left(f_{B} w^{2} \mathrm{~d} \mu\right)^{1 / 2} \leq\left(f_{B} w^{p} \mathrm{~d} \mu\right)^{1 / p} \leq c_{w} f_{B} w \mathrm{~d} \mu .
$$


Therefore, we get

$$
\begin{aligned}
\left(f_{B}\left|w-w_{B}\right| \mathrm{d} \mu\right)^{2} & \leq f_{B}\left(w-w_{B}\right)^{2} \mathrm{~d} \mu \\
& =f_{B}\left(w^{2}-2 w w_{B}+\left(w_{B}\right)^{2}\right) \mathrm{d} \mu \\
& =f_{B} w^{2} \mathrm{~d} \mu-\left(w_{B}\right)^{2} \\
& \leq\left(c_{w}^{2}-1\right)\left(w_{B}\right)^{2} .
\end{aligned}
$$

Now just take square roots to complete the proof.

Lemma 8.2. If $w \in R H_{p}(\mu)$ with constant $c_{w}<5^{1 / p^{\prime}}$ and $p \in(1,2)$, then $w^{p / 2} \in$ $G R_{\varepsilon}(\mu)$ with $\varepsilon=\sqrt{c_{w}^{p^{\prime}}-1}$.

Proof. Let us write $u:=w^{p / 2}$ and $\frac{2}{p}=2 t+(1-t)$ for some $t \in(0,1)$. Then the assumption $w \in R H_{p}(\mu)$ and Hölder's inequality (applied to exponents $\frac{1}{t}$ and $\frac{1}{1-t}$ ) give

$$
\begin{aligned}
f_{B} u^{2} \mathrm{~d} \mu & \leq c_{w}^{p}\left(f_{B} u^{2 / p} \mathrm{~d} \mu\right)^{p} \\
& \leq c_{w}^{p}\left(f_{B} u^{2} \mathrm{~d} \mu\right)^{t p}\left(f_{B} u \mathrm{~d} \mu\right)^{(1-t) p} .
\end{aligned}
$$

This leads to

$$
\left(f_{B} u^{2} \mathrm{~d} \mu\right)^{1 / 2} \leq c_{w}^{p^{\prime} / 2} f_{B} u \mathrm{~d} \mu
$$

which shows that $w^{p / 2} \in R H_{2}(\mu)$. The claim follows once we apply Lemma 8.1

Theorem 8.3. Let $w \in R H_{p}(\mu), 1<p<\infty$, with the defining constant $c_{w}$ close to 1 . Then there exists $q\left(c_{w}\right)>p$ such that $w \in R H_{q}(\mu)$ whenever $p \leq q<q\left(c_{w}\right)$. We have $q\left(c_{w}\right) \rightarrow \infty$ as $c_{w} \rightarrow 1$.

Proof. If $c_{w}$ is close enough to 1 , Lemmas 7.2 , 8.1 and 8.2 are applicable.

Suppose first that $p \geq 2$. Then by Lemma 8.1 we have $w \in G R_{\varepsilon}(\mu)$ with $\varepsilon=\sqrt{c_{w}^{2}-1}$. By Theorem 3.1. $w$ satisfies the weak reverse Hölder inequality whenever $1<q<1+c / \sqrt{c_{w}^{2}-1}$. By Lemma 7.2, $w$ is a doubling weight, further giving $w \in R H_{q}(\mu)$.

Now consider the case $1<p<2$. Then, by Lemma 8.2 we have $w^{p / 2} \in G R_{\varepsilon}(\mu)$ with $\varepsilon=\sqrt{c_{w}^{p^{\prime}}-1}$. Using Theorem 3.1. Hölder's inequality and Lemma 7.2, we get

$$
\left(f_{B} w^{p q / 2} \mathrm{~d} \mu\right)^{1 / q} \leq c f_{2 B} w^{p / 2} \mathrm{~d} \mu \leq c\left(f_{B} w \mathrm{~d} \mu\right)^{p / 2}
$$

whenever $1<q<1+c / \sqrt{c_{w}^{p^{\prime}}-1}$. This implies $w \in R H_{q}(\mu)$ for

$$
1<q<\frac{p}{2}\left(1+c / \sqrt{c_{w}^{p^{\prime}}-1}\right)
$$

provided $c_{w}$ is small enough to guarantee that the upper bound for $q$ is larger than 1 . 
A closing remark is due. While all the embedding theorems in sections 7-9 are known results in the Euclidean space for the Lebesgue measure (see [3], 17], 27] and [31]), it is not obvious if the arguments used in the cited articles work for other measures. Our methods imply that Theorems 6.2 7.3 and 8.3 hold true for arbitrary absolutely continuous (non-doubling) measures in $\mathbb{R}^{n}$. This is a consequence of the result of A. Korenoskyy, A. Lerner and A. Stokolos 20] (the reverse Hölder inequality for Gurov-Reshetnyak weights, in $\mathbb{R}^{n}$ for absolute continuous measures) and our Lemmas 6.1, 7.1, 8.1 and 8.2 (whose simple proofs do not use the doubling property of the underlying measure $\mu$ ). Previously, $A_{p}$ weights, reverse Hölder inequalities and Gehring's lemma for absolutely continuous measures were studied by J. Orobitg and C. Pérez [30] and by J. Martín and M. Milman [25], but our approach gives explicit and asymptotically optimal bounds for the exponents.

\section{ACKNOWLEDGEMENTS}

The first author was supported by the Finnish Academy of Science and Letters, Vilho, Yrjö and Kalle Väisälä Foundation and by the Academy of Finland grant no. 135005. The second author was supported by the Finnish Cultural Foundation. The authors would like to thank J. Kinnunen for discussions.

\section{REFERENCES}

[1] D. Aalto. The discrete maximal operator in metric spaces. J. Anal. Math. 111 (2010), 369390. MR2747071

[2] D. Aalto. Weak $L^{\infty}$ and bmo in metric spaces. (arXiv:0910.1207)

[3] B. Bojarski. Remarks on the stability of reverse Hölder inequalities and quasi-conformal mappings. Ann. Acad. Sci. Fenn. Ser. A I Math. 10 (1985), 89-94. MR802470 (87g:30019)

[4] B. Bojarski. On Gurov-Reshetnyak classes. Bounded Mean Oscillation in complex analysis, University of Joensuu, Publications in sciences, no. 14, p. 21-42, Joensuu, 1989.

[5] B. Bojarski, C. Sbordone and I. Wik. The Muckenhoupt class $A_{1}(\mathbb{R})$. Studia Math. 101(2) (1992), 155-163. MR1149569 (93f:42039)

[6] R. Coifman and G. Weiss. Analyse harmonique non-commutatives surcertains espaces homegènes. Springer-Verlag, Heidelberg, 1971. MR0499948(58:17690)

[7] D. Cruz-Uribe SFO and C. J. Neugebauer. The structure of the reverse Hölder classes. Studia Math. 347 (8) (1995), 2941-2960. MR1308005 (95m:42026)

[8] M. Franciosi. Weighted rearrangements and higher integrability results. Studia Math. 92 (1989), 131-139. MR986944 (90e:42036)

[9] M. Franciosi. On weak reverse integral inequalities for mean oscillations. Proc. Amer. Math. Soc. 113 (1991), 105-112. MR 1068122 (91k:42029)

[10] J. García-Cuerva and J. L. Rubio de Francia. Weighted Norm Inequalities and Related Topics. North Holland, Amsterdam, 1985. MR807149 (87d:42023)

[11] F. W. Gehring. The $L^{p}$-integrability of the partial derivatives of a quasiconformal mapping. Acta Math. 130 (1973), 265-277. MR0402038 (53:5861)

[12] L. G. Gurov. The stability of Lorentz transformations. Estimates for the derivatives. (Russian) Dokl. Akad. Nauk SSSR 220 (1975), 273-276. MR0407778(53:11549)

[13] L. G. Gurov and Yu.G. Reshetnyak. A certain analogue of the concept of a function with bounded mean oscillation. (Russian) Sibirsk. Mat. Z. 17 (1976), 540-546. MR0427565 $(55: 596)$

[14] D. V. Isangulova. The class of mappings with bounded specific oscillation, and integrability of mappings with bounded distortion on Carnot groups. Siber. Math. J. 48, No. 2 (2007), 249-267. MR2330061 (2008e:30029)

[15] D. V. Isangulova. Local stability of mappings with bounded distortion on Heisenberg groups. Siber. Math. J. 48, No. 6 (2007), 984-997. MR2397505(2008m:30023)

[16] T. Iwaniec. On $L^{p}$-integrability in PDE's and quasiregular mappings for large exponents. Ann. Acad. Sci. Fenn. Ser. A I Math. 7 (1982), 301-322. MR686647(84h:30026) 
[17] J. Kinnunen. A stability result on Muckenhoupt's weights. Publ. Math. 42 (1998), 153-163. MR.1628162(99e:42025)

[18] A. A. Korenovskyy. The reverse Hölder inequality, the Muckenhoupt condition, and equimeasurable rearrangements of functions. Russian Acad. Sci. Dokl. Math. 45 (1992), 301-304. MR 1191537(93i:26014)

[19] A. A. Korenovskyy. The connection between mean oscillations and exact exponents of summability of functions (Russian). Mat. Sb. 181 (1990), 1721-1727; translation in Math. USSR-Sb. 71 (1992), 561-567. MR.1099524 (92b:26019)

[20] A. A. Korenovskyy, A. K. Lerner and A. M. Stokolos. A note on the Gurov-Reshetnyak condition. Math. Res. Lett. 9 (5-6) (2002), 579-585. MR.1906061 (2004c:42043)

[21] M. B. Korey. Ideal weights: Doubling and absolute continuity with asymptotically optimal bounds. J. Fourier Anal. Appl. 4 (1998), vol. 4, 491-519. MR1658636 (99m:42032)

[22] R. Korte and O. E. Kansanen Strong $A_{\infty}$-weights are $A_{\infty}$-weights on metric spaces. Rev. Math. Iberoam. 27 (2011) (1), 335-354. MR2815740

[23] O. E. Kansanen. The Gehring lemma in metric spaces. (arXiv:0704.3916)

[24] N. A. Malaksiano. Exact Inclusions of Gehring Classes in Muckenhoupt Classes (Russian). Mat. Zametki 70 (2001), no. 5, 742-750; translation in Math. Notes 70 (2001), no. 5-6, 673-681. MR 1882347 (2002m:30044)

[25] J. Martín and M. Milman. Gehring's lemma for nondoubling measures. Michigan Math. J. 47 (2000), 559-573. MR 1813544 (2002c:42026)

[26] J. Mateu, P. Mattila, A. Nicolau and J. Orobitg. BMO for nondoubling measures. Duke Math. J. 102 (3) (2000), 533-565. MR1756109 (2001e:26019)

[27] T. Mitsis. Embedding $B_{\infty}$ into Muckenhoupt classes. Proc. Amer. Math. Soc. 133 (4) (2004), 1057-1061. MR2117206(2005i:42031)

[28] B. Muckenhoupt. Weighted reverse weak type inequalities for the Hardy-Littlewood maximal function. Pacific. J. Math. 117 (2) (1985), 305-310. MR779926 (86j:42025)

[29] C. J. Neugebauer. The precise range of indices for the $R H_{r}$ and $A_{p}$-classes. (arXiv:9809162)

[30] J. Orobitg and C. Perez. $A_{p}$ weights for nondoubling measures in $\mathbb{R}^{n}$ and applications. Trans. Amer. Math. Soc. 354 (5) (2002), 2013-2033. MR.1881028 (2002k:42044)

[31] A. Politis. Sharp results on the relation between weight spaces and BMO. Ph.D. Thesis, University of Chicago (1995). MR2716561

[32] Yu. G. Reshetnyak. Stability estimates in Liouville's theorem, and the Lp-integrability of the derivatives of quasi-conformal mappings. Siberian Math. J. 17 (1976), 653-674.

[33] J.-O. Strömberg and A. Torchinsky. Weighted Hardy Spaces, vol. 1381 of Lecture Notes in Mathematics. Springer-Verlag, 1989. MR.1011673 (90j:42053)

[34] I. Wik. Note on a theorem by Reshetnyak-Gurov. Studia Math. 86 (1987), 287-290. MR.917054 (89d:26014)

[35] I. Wik. Reverse Hölder inequalities with constant close to 1. Ricerche Mat. 39 (1990), 151-157. MR:1101311 (92g:30028)

Department of Signal Processing and Acoustics, Aalto University School of Electrical Engineering, FI-00076 Aalto University, Finland

E-mail address: daniel.aalto@iki.fi

Department of Mathematics, FI-90014 University of Oulu, Finland

E-mail address: lauri.berkovits@oulu.fi 